Reprogramming goes through a primitive-streaklike state

Expression of the transcription factors OCT3/4, SOX2, KLF4 and c-MYC induces reprogramming of differentiated cells to a pluripotent state (induced pluripotent stem cells (iPSCs)). To understand the mechanisms behind the reprogramming of human cells, Yamanaka and colleagues (Nat. Commun. http://dx.doi. org/10.1038/ncomms4678; 2014) isolated cells at an intermediate stage of reprogramming on the basis of the expression of the surface marker TRA-1-60 and examined how they reach pluripotency. They uncovered the transient activation of the expression of genes characteristic of the mesendoderm, a lineage in the primitive streak in developing embryos, with the upregulation of mesendodermal markers, such as BRACHYURY (T), MIXL1, CER1, LHX1 and EOMES. They observed the presence of active chromatin marks at these promoters as well as an increased level of the protein they encode. Transient activation of these genes happened regardless of the germ-layer origin of the differentiated cells.

The authors then examined the effect of transcription factors linked to primitive streak specification on reprogramming, and found that FOXH1 promotes the maturation of intermediately reprogrammed cells to the pluripotent state. Expression of FOXH1 in the
TRA-1 $-60^{+}$cells enhanced the induction of epithelial markers, consistent with the notion that a mesenchymal-to-epithelial transition state is involved in reprogramming.

Future studies will be required to understand how OCT3/4, SOX2, KLF4 and c-MYC induce the mesendodermal fate during this process.

NLB

\section{The vascular niche directs lymphoma aggressiveness}

The tumour vasculature is known to have tumour-promoting effects, such as increased tumour growth and invasion. Rafii and colleagues now show that tumour endothelial cells participate in an intricate crosstalk with lymphoma cells to promote lymphoma cell invasiveness and resistance to chemotherapy (Cancer Cell 25, 350-365; 2014).

The authors devised an endothelial and lymphoma cell co-culture system followed by in vivo mouse experiments and transcriptomic profiling to demonstrate that endothelial cells promote expansion of an aggressive and chemoresistant lymphoma cell subpopulation. A series of elegant in vitro and in vivo experiments demonstrated that FGF4 produced by B cell lymphoma cells activated FGFR1 signalling in endothelial cells, leading to the upregulation of the Notch ligand Jag1. Endothelial cells expressing Jag-1 were in turn shown to

\title{
Mitotic suppression of repair prevents telomere fusions
}

Repair of DNA double-strand breaks (DSBs) requires accumulation of the ubiquitin ligase RNF8 followed by the repair factors 53BP1 and BRCA1 at break sites. Recruitment of these proteins and subsequent repair is inhibited in mitotic cells, but it has been unclear how and why. Durocher and colleagues reveal that RNF8 recruitment is controlled by the mitotic kinases CDK1 and PLK1, and that this is important for the maintenance of genomic stability (Science http://doi.org/r8g; 2014).

RNF8 is normally recruited through binding to MDC1 but the authors found that CDK1mediated phosphorylation of RNF8 on Thr 198 disrupts its interaction with RNF8. In RNF8-depleted cells, restoration of a non-phosphorylatable RNF8 mutant restored irradiation-induced RNF8 foci in mitosis, whereas a phospho-mimicking version prevented foci formation in interphase. Reversing this phosphorylation event was sufficient to restore the recruitment of BRCA, but not that of 53BP1, to mitotic DSB sites. The authors then identified PLK1 and CDK1 phosphorylation sites in 53BP1, and demonstrated by mutagenesis that PLK1- and CDK1-mediated phosphorylation of 53BP1 prevents its recruitment to DSBs.

Cells expressing non-phosphorylatable mutants of both RNF8 and 53BP1 were extremely sensitive to ionizing radiation because of impaired whole-chromosome segregation associated with telomere fusions, a process mediated by the repair machinery. Thus mitotic chromosomes may be sensitive to telomere fusions, explaining why DSB repair must be suppressed in mitosis.

activate Notch2 signalling in neighbouring lymphoma cells to drive their expansion, lymphomagenesis, extranodal invasiveness and resistance to chemotherapy, as shown using an $E \mu-M y c$ lymphoma mouse model. Disrupting the crosstalk of endothelial and lymphoma cells by genetic inactivation of Fgfr 1 or Jag1 in endothelial cells reduced tumour growth and increased mouse survival and sensitivity to chemotherapeutic agents.

These findings elucidate a non-cell-autonomous mechanism through which lymphoma cells foster their own expansion and aggressiveness, by instigating the formation of a protumorigenic vascular niche.

AIZ

\section{ESCRTing intraluminal vesicle formation}

Endosomal sorting complex required for transport (ESCRT) proteins are involved in the formation of intraluminal vesicles (ILVs) at multivesicular bodies (MVBs). Teis and colleagues now reveal a role for the ESCRTassociated AAA-ATPase Vps4 in ILV budding (J. Cell Biol. http://doi.org/r8j; 2014).

ESCRT-III proteins wrap around the necks of forming ILVs to promote membrane scission. The ESCRT-III complex is then disassembled and recycled by Vps4, which interacts with the ESCRT-III proteins Vps2, Did2, Vps20, Vps24 and Snf7. The authors expressed Vps4interaction-deficient ESCRT mutants in yeast, and found that mutations in Vps2 and Snf7 reduced Vps4 association with the ESCRT-III complex and blocked complex disassembly.

Surprisingly, the Vps2 and Snf7 mutants also blocked cargo sorting into the vacuole lumen. In addition, ILVs in Vps2- or Snf7-mutant yeast were larger but formed less frequently than their wild-type counterparts. These observations point to a potential role for Vps4 in ILV generation. The authors propose that Vps4 remodels the ESCRTIII complex to promote membrane deformation, membrane scission and ILV biogenesis. Future experiments aimed at understanding the precise role for Vps4 in these processes, and whether it is required for other ESCRT-III-driven scission events, will provide further support for these intriguing observations. EJC

By Nathalie Le Bot, Emily J. Chenette, Christina Karlsson Rosenthal and Alexia-Ileana Zaromytidou 